\title{
Stellar wobble caused by a binary system: Can it really be mistaken as an extra-solar planet?
}

\author{
M. H. M. Morais ${ }^{1}$ and A. C. M. Correia ${ }^{2,3}$ \\ ${ }^{1}$ Centro de Física Computacional, Universidade de Coimbra, 3004-516 Coimbra, Portugal \\ e-mail: hmorais@mat.uc.pt \\ 2 Departamento de Física, Universidade de Aveiro, Campus de Santiago, 3810-193 Aveiro, Portugal \\ e-mail: correia@ua.pt \\ 3 IMCCE, CNRS-UMR8028, Observatoire de Paris, UPMC, 77 avenue Denfert-Rochereau, 75014 Paris, France
}

Received 4 August 2008 / Accepted 25 September 2008

\begin{abstract}
The traditional method for detecting extra-solar planets relies on measuring a small stellar wobble which is assumed to be caused by a planet orbiting the star. Recently, it has been suggested that a similar stellar wobble could be caused by a close binary system. Here we show that, although the effect of a close binary system can at first sight be mistaken as a planetary companion to the star, more careful analysis of the observational data should allow us to distinguish between the two effects.
\end{abstract}

Key words. techniques: radial velocities - methods: observational - celestial mechanics - stars: planetary systems stars: binaries: general

\section{Introduction}

Over the past decade up to 290 extra-solar planets were discovered using the wobble method (i.e. measuring the motion of the parent star around the planet-star's center of mass). This represents about $95 \%$ of the total extra-solar planets known to date ${ }^{1}$. However, this is an indirect method in which the presence of a planet is inferred from the detection of the radial velocity variations. Therefore, in cases where the presence of the planet can not be confirmed by other methods we may ask if these variations could be due to another dynamical effect. In particular, when a planet is detected in a system already hosting a companion (such as another planet or a star), we must be cautious when we analyze the data, because different configurations of bodies can lead to similar radial velocity variations.

The present paper is motivated by the work of Schneider \& Cabrera (2006) who studied a triple system composed of a binary star system with equal masses orbiting a third star (assumed massless). They concluded that the binary system will cause a wobble in the star's motion that could mimic the presence of a planet companion. Therefore, they suggest that each extra-solar planet detection which relies on the measurement of this wobble must be carefully checked for the presence of possible nearby unresolved binaries.

Unfortunately the study in Schneider \& Cabrera (2006) was restricted to the case of binary systems composed of stars with equal masses, while a multitude of three-body configurations can be imagined. In particular, we may wonder about the effect of a giant satellite orbiting an already detected Jupiter-like extra-solar planet. Moreover, the conclusions in Schneider \& Cabrera (2006) were derived in the framework of the restricted

1 The Extrasolar Planets Encyclopedia. http://exoplanet.eu/ three-body problem and we may also question the validity of this approximation. Additionally, we found that their work contains several inaccurate conclusions.

Our goal is to correctly model the effect of a binary system on a nearby star without making any assumptions on the masses of this triple system. In Sect. 2 we briefly review the wobble method for detecting planets. In Sect. 3 we derive simple expressions for the radial velocity of a star under the presence of a nearby binary system and for the orbital parameters of a planet mimicked by this effect. In Sect. 4 we give some examples of binary systems and present numerical simulations to test our theory. Finally, the last section is devoted to a discussion of the results.

\section{The wobble method}

We quickly review the wobble method for detecting extra-solar planets (see for instance Hilditch 2001). Assume that we are observing a star with mass $M_{\star}$, which has a planet companion with mass $M_{\mathrm{p}}$. A possible observation reference frame is depicted in Fig. 1a, where we have the $\hat{\boldsymbol{x}}$ and $\hat{z}$ axes along the line of nodes and the line of sight, respectively. The orbital plane $(\hat{\boldsymbol{\imath}}, \hat{\boldsymbol{J}})$ is depicted in Fig. 1b, where $\hat{\boldsymbol{\imath}} \equiv \hat{\boldsymbol{x}}$. In this last frame the position of the star/planet can be written as

$\boldsymbol{r}=X \hat{\boldsymbol{\imath}}+Y \hat{\boldsymbol{\jmath}}=r \cos (\varpi+f) \hat{\boldsymbol{\imath}}+r \sin (\varpi+f) \hat{\boldsymbol{j}}$,

where $r$ is the orbital radius, $f$ the true anomaly, and $\varpi$ the longitude of the periastron.

Since $\hat{\boldsymbol{\imath}} \equiv \hat{\boldsymbol{x}}$, the $\hat{\boldsymbol{j}}$ axis is simply obtained with a rotation of the $\hat{\boldsymbol{y}}$ axis by an angle $I$, the inclination of the orbital plane measured with respect to the plane $(\hat{\boldsymbol{x}}, \hat{\boldsymbol{y}})$. Thus, we may write 


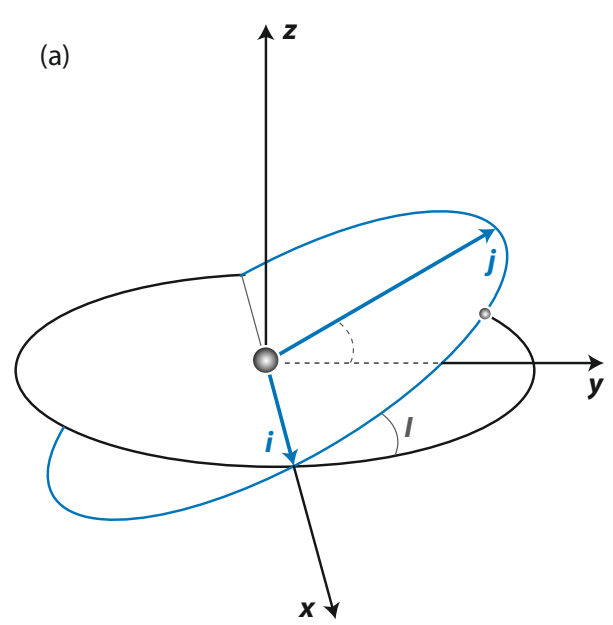

(b)



Fig. 1. Reference frames. The plane defined by $(\hat{\boldsymbol{x}}, \hat{\boldsymbol{y}})$ is the plane of the sky and the $\hat{z}$ axis is along the line of sight. The plane $(\hat{\boldsymbol{\imath}}, \hat{\boldsymbol{J}})$ is the orbital plane, $\varpi$ the argument of the periastron and $f$ the true anomaly. For simplicity we chose the $\hat{\boldsymbol{x}}$ axis along the line of nodes $(\hat{\boldsymbol{\imath}} \equiv \hat{\boldsymbol{x}})$.

$z=Y \sin I$, where $z$ is the position along the $\hat{z}$ axis. The velocity along the line of sight is then

$$
\begin{aligned}
\dot{z} & =\dot{Y} \sin I \\
& =(\dot{r} \sin (\varpi+f)+r \dot{f} \cos (\varpi+f)) \sin I \\
& =K_{z}[\cos (\varpi+f)+e \cos \varpi],
\end{aligned}
$$

where $e$ is the orbital eccentricity and $K_{z}=n a_{\mathrm{p}} \sin I / \sqrt{1-e^{2}}$, with $n$ the mean motion, and $a_{\mathrm{p}}$ the semi-major axis of the orbit.

The radial velocity of the star is the projection of the velocity with respect to the center of mass (CM) along the line of sight. In the particular case of a circular orbit, we have $e=0$ and $\dot{f}=n$, thus

$$
V_{r}=\frac{M_{\mathrm{p}}}{M_{\star}+M_{\mathrm{p}}} \dot{z}=K \cos (\varpi+n t),
$$

where $t$ is the time and the amplitude

$K=\frac{M_{\mathrm{p}}}{M_{\star}+M_{\mathrm{p}}} n a_{\mathrm{p}} \sin I$.

If we analyze the Fourier spectrum of the star's radial velocity we will see that this will exhibit a peak at the frequency $n$ and the amplitude $K$ provides a lower limit for the planet's mass. This method of detection of extra-solar planets is also known as the wobble method (since the planet causes the star to orbit or wobble about their common CM).

Notice also that when more than one companion to the star is present (another planet or star), the total radial velocity will be given by a sum of the individual contribution from each body,

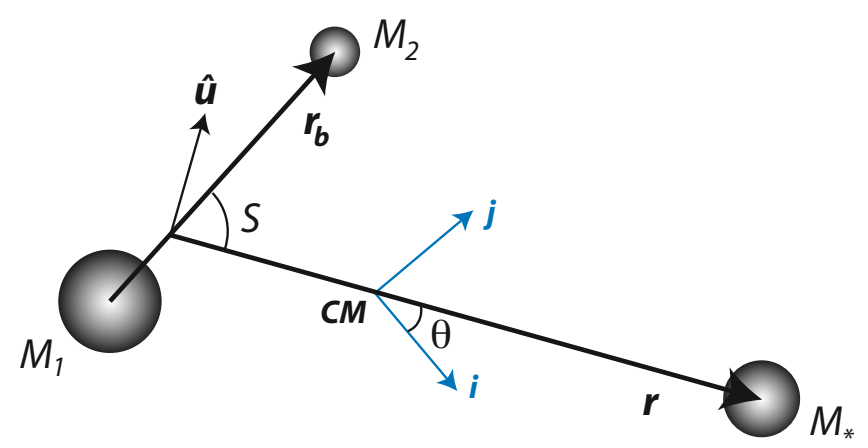

Fig. 2. Jacobi coordinates $\left(\boldsymbol{r}_{\mathrm{b}}, \boldsymbol{r}\right)$. The system is composed by a star with mass $M_{\star}$ and a binary with masses $M_{1}$ and $M_{2}$. We assume co-planar motion and the frame $(\hat{\boldsymbol{v}}, \hat{\boldsymbol{J}})$ is the same from Fig. 1. $\hat{\boldsymbol{u}}$ is a unit vector normal to $\boldsymbol{r}, S=\angle\left(\boldsymbol{r}, \boldsymbol{r}_{\mathbf{b}}\right)$ and $\theta=\angle(\hat{\boldsymbol{\imath}}, \boldsymbol{r})$.

$V_{r}=\sum_{k} M_{k} \dot{z}_{k} / M_{\mathrm{Tot}}$, where $M_{\mathrm{Tot}}=M_{\star}+\sum_{k} M_{k}$ is the total mass of the system. The amplitude due to a specific companion is then:

$K_{\mathrm{p}}=\frac{M_{\mathrm{p}}}{M_{\star}+\sum_{k} M_{k}} n a_{\mathrm{p}} \sin I$.

\section{The model and its predictions}

We will work in the framework of the planar general three body problem: we assume that a star with mass $M_{\star}$ moves in the same orbital plane as the binary system composed of two bodies with masses $M_{1}$ and $M_{2}$ (Fig. 2).

\subsection{Equations of motion}

In order to obtain the equations of motion we will use Jacobi canonical coordinates, which are, respectively, the inter-binary distance, $\boldsymbol{r}_{\mathbf{b}}$, and the distance, $\boldsymbol{r}$, from the star $M_{\star}$ to the binary's CM (see Fig. 2). We will additionaly assume that $\left|\boldsymbol{r}_{\mathbf{b}}\right| \ll$ $|\boldsymbol{r}|$. Thus, to the second order in $\rho=\left|\boldsymbol{r}_{\mathbf{b}}\right| /|\boldsymbol{r}| \ll 1$, the Hamiltonian of the system can be expressed as (e.g. Henrard 1979; Murray \& Dermott 1999):

$$
\begin{aligned}
H= & \frac{1}{2} \frac{\left|p_{\mathrm{b}}\right|^{2}}{\mu}+\frac{1}{2} \frac{|\boldsymbol{p}|^{2}}{\mu_{\star}}-G \frac{M_{1} M_{2}}{r_{\mathrm{b}}}-G \frac{M M_{\star}}{r} \\
& -G \frac{M_{1} M_{2} M_{\star}}{r M} \rho^{2} \frac{1}{2}\left(3 \cos ^{2} S-1\right),
\end{aligned}
$$

where $M=M_{1}+M_{2}$ is the total mass of the binary, $S=\angle\left(\boldsymbol{r}, \boldsymbol{r}_{\mathbf{b}}\right)$,

$\mu=\frac{M_{1} M_{2}}{M} \quad$ and $\quad \mu_{\star}=\frac{M M_{\star}}{M+M_{\star}}$

are the reduced masses, and $\boldsymbol{p}_{\mathrm{b}}=\mu \dot{\boldsymbol{r}}_{\mathrm{b}}$ and $\boldsymbol{p}=\mu_{\star} \dot{\boldsymbol{r}}$ the conjugate momenta of $\boldsymbol{r}_{\mathrm{b}}$ and $\boldsymbol{r}$, respectively. In this framework, since the Jacobi coordinates are canonical, the equations of motion are easily obtained as

$\dot{\boldsymbol{p}}_{\mathrm{b}}=-\frac{\partial H}{\partial \boldsymbol{r}_{\mathrm{b}}}$ and $\dot{\boldsymbol{p}}=-\frac{\partial H}{\partial \boldsymbol{r}}$.

\subsection{Motion of the binary}

From Eq. (8) the equation of motion for $\boldsymbol{r}_{b}$ is

$\ddot{\boldsymbol{r}}_{\mathrm{b}}=-G \frac{M}{r_{b}^{3}} \boldsymbol{r}_{\mathbf{b}}+G \frac{M_{\star}}{r^{3}}\left(3 \rho \cos S \boldsymbol{r}-\boldsymbol{r}_{\mathbf{b}}\right)$. 
We can neglect the second term in Eq. (9) if $\rho^{3} \ll M / M_{\star}$, which is always true if we choose a "stable binary", i.e. if $M_{1} \geq M_{2}$, such that

$\rho=\alpha\left(\frac{M_{1}}{3 M_{\star}}\right)^{1 / 3}$,

where $\alpha \lesssim 0.5$ (Markellos \& Roy 1981). Therefore, the 0th order solution for $\boldsymbol{r}_{\mathbf{b}}$ is a Keplerian ellipse with constant semi-major axis $a_{\mathrm{b}}$ and frequency

$\omega=\sqrt{\frac{G M}{a_{b}^{3}}}$.

\subsection{Motion of the star}

From Eq. (8) the equation of motion for $\boldsymbol{r}$ is

$$
\begin{aligned}
\ddot{\boldsymbol{r}}= & -G \frac{\left(M+M_{\star}\right)}{r^{3}} \times \\
& {\left[\left(1+\frac{\mu}{M} \frac{\rho^{2}}{2}\left(-3+15 \cos ^{2} S\right)\right) \boldsymbol{r}-\frac{\mu}{M} 3 \rho \cos S \boldsymbol{r}_{\mathbf{b}}\right] . }
\end{aligned}
$$

We can neglect the 2nd and 3rd terms in Eq. (12) if

$\rho^{2} \frac{\mu}{M} \ll 1$.

Since $\mu / M \leq 1 / 4$ and from Eq. (10) $\rho^{2} \ll 1$, this is always true. Therefore, the 0th order solution for $\boldsymbol{r}$ is also a Keplerian ellipse with constant semi-major axis $a$ and frequency

$\Omega=\sqrt{\frac{G\left(M+M_{\star}\right)}{a^{3}}}$,

i.e., the motion of $M_{\star}$ is to a first approximation a Keplerian orbit about a mass $M$ located at the binary's CM. Furthermore, from Eqs. (11) and (14) we see that in general $\Omega \ll \omega$ since $a_{\mathrm{b}} \ll a$ (this is true unless $M_{\star}$ becomes too large).

\subsection{Stellar wobble}

Now, we can obtain an approximation for the relative motion of $M_{\star}$ by replacing the 0th order approximations for $\boldsymbol{r}_{\mathbf{b}}$ and $\boldsymbol{r}$ in Eq. (12). We assume that these 0th order solutions are circular orbits, thus

$\boldsymbol{r}=a \hat{\boldsymbol{r}}$,

$\boldsymbol{r}_{\mathbf{b}}=a_{\mathrm{b}} \cos S \hat{\boldsymbol{r}}+a_{\mathrm{b}} \sin S \hat{\boldsymbol{u}}$,

$S=S_{0}+(\omega-\Omega) t$,

where $\hat{\boldsymbol{r}}$ is the versor of $\boldsymbol{r}, \hat{\boldsymbol{u}}$ is the unit vector orthogonal to $\hat{\boldsymbol{r}}$ and $S_{0}$ is an initial phase (Fig. 2).

In order to compute the radial velocity, we are interested in obtaining the equation of motion for $M_{\star}$ in barycentric coordinates. The distance of the star to the $\mathrm{CM}$ is

$r_{\star}=\frac{M}{M+M_{\star}} r$.

We hence have

$$
\begin{gathered}
\ddot{\boldsymbol{r}}_{\star}=-G \frac{M}{a^{2}}\left[\left(1+\frac{\mu}{M} \rho^{2}\left(\frac{3}{4}+\frac{9}{4} \cos 2 S\right)\right) \hat{\boldsymbol{r}}\right. \\
\left.-\frac{\mu}{M} \rho^{2} \frac{3}{2} \sin 2 S \hat{\boldsymbol{u}}\right],
\end{gathered}
$$

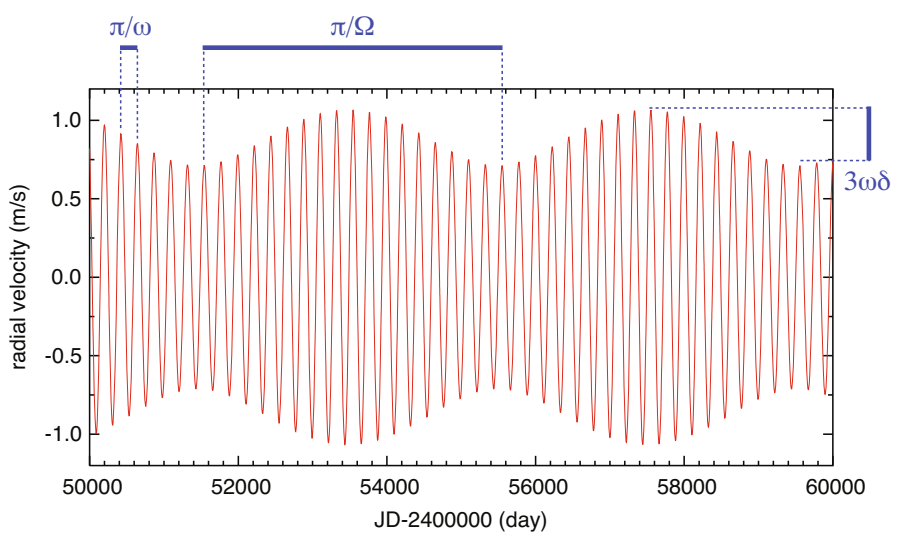

Fig. 3. Radial velocity of a star due to the binary wobble around its own CM (Eq. (25)). The orbital parameters are from Ex.\#3, Table 4.

with $\rho=a_{\mathrm{b}} / a \ll 1$. Notice that if we chose $M_{\star}=0$ and $M_{1}=$ $M_{2}$ (binary with equal masses) in Eq. (19) we recover the same equation of motion obtained by Schneider \& Cabrera (2006).

In Appendix A we determine the solution to Eq. (19) in the inertial frame $(\hat{\boldsymbol{\imath}}, \hat{\boldsymbol{j}})$ with coordinates $\boldsymbol{r}_{\star}=(X, Y)$, that is:

$\left(\begin{array}{l}X \\ Y\end{array}\right)=\left(\begin{array}{c}a_{\star} \cos \theta \\ a_{\star} \sin \theta\end{array}\right)+\left(\begin{array}{cc}\sin \theta & \cos \theta \\ -\cos \theta & \sin \theta\end{array}\right)\left(\begin{array}{c}3 \delta \sin 2 S \\ 4.5 \delta \cos 2 S\end{array}\right)$,

with $a_{\star} \approx a M /\left(M+M_{\star}\right), \theta=\theta_{0}+\Omega t$ and

$\delta=\frac{\mu}{8 M}\left(\frac{a_{\mathrm{b}}}{a}\right)^{4} a_{\mathrm{b}}$

This solution has two components, the first one caused by the motion of the star around the binary's CM and the second one is the stellar wobble caused by the the movement of the two bodies of the binary around its own CM.

According to our choice of reference frame (Fig. 1), the radial velocity of the $\operatorname{star} M_{\star}$ is given by $V_{r}=\dot{Y} \sin I$, that is, we can obtain it directly from the $Y$ coordinate. From Eq. (20) we have:

$Y=a_{\star} \sin \theta-3 \delta \sin 2 S \cos \theta+4.5 \delta \cos 2 S \sin \theta$,

Because $\dot{S}=\omega-\Omega$ and $\dot{\theta}=\Omega$, and since we assume $\Omega \ll \omega$, the velocity along $\hat{\boldsymbol{y}}$ is then

$\dot{Y}=a_{\star} \Omega \cos \theta-6 \omega \delta \cos 2 S \cos \theta-9 \omega \delta \sin 2 S \sin \theta$.

The first term in Eq. (23) is due to the slow motion of the star around the binary's CM. The second and third terms are due to the binary wobble. These last two terms can be seen as a composition of two periodic signals, one fast with frequency $2 \omega-2 \Omega$ and another much slower with frequency $\Omega$. The global effect of the binary wobble corresponds to a signal of period $\pi /(\omega-\Omega)$ with an amplitude modulation of $3 \omega \delta$ and period $\pi / \Omega$ (Fig. 3).

\subsection{Mimicking a planet}

Even though the radial velocity of a star perturbed by a binary system is not given by a single periodic signal (Fig. 3), we may ask under what circumstances this signal can be misinterpreted as a planet orbiting the star. We can rewrite Eq. (23) in the same format as Eq. (3), i.e.

$V_{r}=K_{0} \cos \left(\theta_{0}+\Omega t\right)+V_{r}^{b}$, 
Table 1. Planets detected in close binary systems and the respective parameters $\left(a_{\mathrm{b}}, M_{2}\right)$ of the stellar companion that can mimic them.

\begin{tabular}{|c|c|c|c|c|c|c|c|c|}
\hline System name & $M_{*}\left(M_{\odot}\right)$ & $M_{1}\left(M_{\odot}\right)$ & $M_{\mathrm{p}}\left(M_{\text {Jup }}\right)$ & $a(\mathrm{AU})$ & $a_{\mathrm{p}}(\mathrm{AU})$ & $M_{2}\left(M_{\odot}\right)$ & $a_{\mathrm{b}}(\mathrm{AU})$ & $\alpha$ \\
\hline GJ $86 \mathrm{~A}^{1}$ & 0.79 & $\sim 0.5$ & 4.01 & $\sim 18$ & 0.11 & $3.4 \times 10^{3}$ & 2.85 & 0.27 \\
\hline$\gamma$ Cep A $^{2}$ & 1.40 & 0.41 & 1.60 & 20.2 & 2.04 & 3.81 & 4.68 & 0.50 \\
\hline HD $41004 \mathrm{~A}^{3}$ & 0.7 & 0.4 & 2.54 & 23 & 1.31 & 11.9 & 5.41 & 0.41 \\
\hline HD $41004 \mathrm{~B}^{3}$ & 0.4 & 0.7 & 18.37 & 23 & 0.018 & $4.9 \times 10^{5}$ & 3.06 & 0.16 \\
\hline HD $196885 \mathrm{~A}^{4}$ & 1.33 & $\sim 0.6$ & 2.96 & $\sim 23$ & 2.63 & 2.93 & 5.78 & 0.47 \\
\hline HD $19994 A^{5}$ & 1.34 & 0.3 & 1.68 & $\sim 100$ & 1.42 & $6.2 \times 10^{2}$ & 17.4 & 0.41 \\
\hline
\end{tabular}

References: ${ }^{1}$ Queloz et al. (2000); Lagrange et al. (2006); ${ }^{2}$ Neuhäuser et al. (2007); ${ }^{3}$ Zucker et al. (2004); ${ }^{4}$ Correia et al. (2008). ${ }^{5}$ Mayor et al. (2004).

where $K_{0}=a_{\star} \Omega \sin I$, and

$V_{r}^{b}=K_{1} \cos \left(\varpi_{1}+n_{1} t\right)+K_{2} \cos \left(\varpi_{2}+n_{2} t\right)$

with

$K_{1}=-\frac{15}{2} \omega \delta \sin I \quad$ and $\quad K_{2}=\frac{3}{2} \omega \delta \sin I$,

$n_{1}=2 \omega-3 \Omega$ and $n_{2}=2 \omega-\Omega$,

and

$\varpi_{1}=2 S_{0}-\theta_{0}$ and $\varpi_{2}=2 S_{0}+\theta_{0}$.

The 1st term in Eq. (24) represents the slow two-body motion around a body with mass $M$ located at the binary's CM. We recall that we may not know about the presence of the binary (since one or even both components may be unresolved) and this term allows us to identify only a single component.

On the other hand, $V_{r}^{b}$ is the radial velocity due to the binary wobble around its own CM and can be identified as two planets on circular orbits around the star. Hence, contrarily to Schneider \& Cabrera (2006), we conclude that the stellar wobble caused by the presence of the binary mimics not one, but two planets, although with very close orbital periods. As the two frequencies $n_{1}$ and $n_{2}$ are very close, in some situations the two signals can be confounded and the observer can erroneously believe to have found a planetary companion to the star.

This will be the case if our instrument is at the limit of its resolution: since $\left|K_{1}\right|=5\left|K_{2}\right|$, the trace of the second "planet" is harder to detect. Therefore, if our spectral resolution is close to the detection limit of the stellar wobble, we can notice the presence of the first "planet", but we will miss the second one.

By applying Kepler's 3rd law, we obtain for the semi-major axis of this fake planet,

$a_{\mathrm{p}}=\frac{\left(G M_{\star}\right)^{1 / 3}}{n_{1}^{2 / 3}} \approx\left(\frac{M_{\star}}{4 M}\right)^{1 / 3} a_{\mathrm{b}}$,

and for the planet's mass, we replace $K_{1}$ from Eq. (26) in Eq. (5):

$\frac{M_{\mathrm{p}}}{M+M_{\star}}=\frac{15}{2} \frac{\delta}{a_{\mathrm{p}}} \frac{\omega}{n_{1}} \approx \frac{15}{32} \frac{\mu}{M}\left(\frac{a_{\mathrm{b}}}{a}\right)^{4} \frac{a_{\mathrm{b}}}{a_{\mathrm{p}}}$.

Notice that when $M_{1}=M_{2}$ our estimate for the planet's mass is different from what was obtained by Schneider \& Cabrera (2006). Additionally, we also conclude that the planet evolves in a circular orbit (while $e=0.745$ from Eq. (4) of Schneider \& Cabrera 2006).

\subsection{Distinguishing between a binary and a planet}

The situation described above is very likely and we may expect to interpret erroneously the signal of a binary as a planet. However, the radial velocity wobble produced by a binary (Eq. (25)), is different from the radial velocity produced by a single planet (Eq. (3)) and therefore we may be able to distinguish between the two situations.

Indeed, if we acquire data for long enough time (so that we can constrain well $\Omega$ ) and if the precision of the instrument is at the limit of detection of the amplitude $K_{2}$, the observer will be able to notice the presence of the two periodic signals $n_{1}$ and $n_{2}$ (Eq. (27)) and therefore realize that the star is undergoing the perturbation from a binary and not from a planet.

Since the radial velocity of the binary (Eq. (25)) is the same as the signal produced by two planets in circular orbits, the observer can also believe that two planets have been found (instead of the binary). However, as long as $\Omega \ll \omega$, the two orbital frequencies $n_{1}$ and $n_{2}$ are very close and probably the orbits of the two planets cannot be stable.

For higher $\Omega$ values, the system may become stable (although the approximation $\Omega \ll \omega$ may not be valid) and the hypothesis of existence of two planets cannot be excluded. Nevertheless, in the case of a binary, the frequencies $\left(n_{1}, n_{2}\right)$ and the amplitudes $\left(K_{1}, K_{2}\right)$ are not independent (Eqs. (27) and (26)) and the following relations must be verified:

$n_{2}-n_{1}=2 \Omega$ and $\left|K_{1}\right|=5\left|K_{2}\right|$.

\subsection{What kind of binary can mimic a planet?}

In Sect. 3.5 we saw that under some particular conditions, the variations in the star's radial velocity resulting from the binary wobble can be misinterpreted as a planet. Thus, if we detect a planet and we do not know about the presence of the binary system (e.g. if $M_{2}$ is unresolved) we can ask the following question: what are the parameters of an hypothetical binary system that can mimic this planet? More precisely, if we know $M_{\star}, M_{1}$, $a, M_{\mathrm{p}}$ and $a_{\mathrm{p}}$, what are the values of $M_{2}$ and $a_{\mathrm{b}}$ ? These can be obtained by inverting Eqs. (29) and (30):

$$
\begin{aligned}
a_{\mathrm{b}} & =\frac{(G M)^{1 / 3}}{\left(\frac{1}{2} n_{1}+\frac{3}{2} \Omega\right)^{2 / 3}} \approx\left(\frac{4 M}{M_{\star}}\right)^{1 / 3} a_{\mathrm{p}}, \\
\mu\left(1+\frac{M_{\star}}{M}\right) & =\frac{16}{15} \frac{n_{1}}{\omega} \frac{a_{\mathrm{p}}}{a_{\mathrm{b}}}\left(\frac{a}{a_{\mathrm{b}}}\right)^{4} M_{\mathrm{p}} \\
& \approx 0.212\left(\frac{M_{\star}}{M}\right)^{5 / 3}\left(\frac{a}{a_{\mathrm{p}}}\right)^{4} M_{\mathrm{p}} .
\end{aligned}
$$

In Table 1 we list all the currently known planets in close binary systems $(a<100 \mathrm{AU})$ and estimate the corresponding 
companions' parameters $\left(M_{2}\right.$ and $\left.a_{\mathrm{b}}\right)$ that can mimic that planet. Three of the cases provide unrealistic binary systems with very high values of $M_{2}$. The remaining three cases are acceptable ( $\gamma$ Cep A, HD 41004 A, and HD 196885 A) but these binary systems are likely to be unstable as $\alpha$ is near the stability limit (see Eq. (10)). On the other hand, the values of $M_{2}$ in these three cases are in the mass range of either black-holes or very bright stars (which should be detectable). Therefore, it is unlikely that these planets can be the result of a binary wobble.

\section{Examples}

In Sect. 3 we obtained the radial velocity wobble of a star that is perturbed by a binary system. We subsequently derived theoretical expressions that allow us to determine the orbital parameters of a planet that can, in some circumstances, be mimicked by those variations. In this section we will present some concrete examples of binary systems together with the orbital parameters of the planets that they can mimic. We also obtain the amplitude of the peaks in the radial velocity curve since these give an indication of the instrument precision needed to detect the planet and to distinguish between the planet and binary.

\subsection{Theory versus numerical integrations}

In order to test the theoretical predictions of our model (Sect. 3), we performed some numerical simulations of three-body systems (Table 4). Here we present in detail a case (Ex.\#3, Table 4) for which the current precision in the radial velocity measurements $(\sim 1 \mathrm{~m} / \mathrm{s})$ could lead to the erroneous announcement of a planet.

The hypothetical system is formed by a triplet of main sequence stars with different spectral types, $\mathrm{G}, \mathrm{K}$ and $\mathrm{M}$, and masses $M_{\star}=1.00 M_{\odot}, M_{1}=0.70 M_{\odot}$ and $M_{2}=0.35 M_{\odot}$, respectively. The smaller $\mathrm{K}$ and $\mathrm{M}$ stars form a binary system on a circular orbit with $a_{\mathrm{b}}=1.1 \mathrm{AU}$. The G-star is in a wider circular orbit around the close binary's CM, with $a=10$ AU. Note that the M star is much fainter than the $\mathrm{K}$ or $\mathrm{G}$ stars hence it represents the unresolved component of the binary. We also suppose that the system is co-planar and perpendicular to the line of sight (that is, $\sin I=1$ ).

We numerically integrated this stellar system and computed the radial velocity of the G-star, the brightest body in the system. In Table 2 we list 50 simulated observational data points for a time span of about 10.5 years (from Feb. 2004 to Sep. 2014). We supposed that the data was acquired with a precision of $\sim 0.8 \mathrm{~m} / \mathrm{s}$, identical to the HARPS spectrograph on the ESO 3.6-m telescope at La Silla (Chile), currently the most precise instrument in operation since the beginning of 2004 (Mayor et al. 2003).

From Eqs. (11) and (14) we compute for this system:

$\frac{2 \pi}{\omega}=411.23$ day and $\frac{2 \pi}{\Omega}=8067.0$ day .

The observational data do not fully cover the orbital period of the G-star around the binary's CM, but we will be nevertheless able to estimate it, since we are assuming a high level of precision in the data.

According to our model predictions (Sect. 3.5), the radial velocity of the G-star can be decomposed in three sinusoidal terms (Eq. (24)). One of large amplitude, $K_{0}=6.907 \mathrm{~km} \mathrm{~s}^{-1}$, but varying slowly with period $2 \pi / \Omega$, and the other two terms varying much faster with periods (Eq. (27))

$\frac{2 \pi}{n_{1}}=222.64$ day and $\frac{2 \pi}{n_{2}}=210.99$ day,
Table 2. Simulated radial-velocity data for a triple system of stars with $M_{\star}=1.00 M_{\odot}, M_{1}=0.70 M_{\odot}, M_{2}=0.35 M_{\odot}, a=10.0 \mathrm{AU}$ and $a_{\mathrm{b}}=1.1 \mathrm{AU}$. The 50 points are generated for a period of about $10.5 \mathrm{yr}$.

\begin{tabular}{|c|c|c|}
\hline $\begin{array}{c}\text { Julian date } \\
\text { [T-2 400 000] }\end{array}$ & $\begin{array}{c}R \boldsymbol{R} \\
{\left[\mathrm{km} \mathrm{s}^{-1}\right]}\end{array}$ & $\begin{array}{c}R V \text { error } \\
{\left[\mathrm{km} \mathrm{s}^{-1}\right]}\end{array}$ \\
\hline 53063. & 9.0534 & 0.0014 \\
\hline 53093. & 9.2167 & 0.0007 \\
\hline 53151. & 9.5290 & 0.0011 \\
\hline 53191. & 9.7458 & 0.0010 \\
\hline 53207. & 9.8315 & 0.0009 \\
\hline 53252. & 10.0764 & 0.0009 \\
\hline 53291. & 10.2879 & 0.0015 \\
\hline 53435. & 11.0649 & 0.0008 \\
\hline 53450. & 11.1445 & 0.0014 \\
\hline 53520. & 11.5204 & 0.0007 \\
\hline 53564. & 11.7537 & 0.0012 \\
\hline 53618. & 12.0344 & 0.0012 \\
\hline 53654. & 12.2186 & 0.0009 \\
\hline 53809. & 13.0006 & 0.0011 \\
\hline 53869. & 13.2901 & 0.0019 \\
\hline 53892. & 13.3982 & 0.0009 \\
\hline 53934. & 13.5962 & 0.0008 \\
\hline 53986. & 13.8352 & 0.0009 \\
\hline 54029. & 14.0281 & 0.0011 \\
\hline 54161. & 14.5877 & 0.0007 \\
\hline 54206. & 14.7691 & 0.0008 \\
\hline 54306. & 15.1462 & 0.0007 \\
\hline 54349. & 15.3000 & 0.0009 \\
\hline 54360. & 15.3383 & 0.0009 \\
\hline 54544. & 15.9165 & 0.0010 \\
\hline 54570 . & 15.9898 & 0.0009 \\
\hline 54651. & 16.2000 & 0.0008 \\
\hline 54954. & 16.7522 & 0.0007 \\
\hline 55000 . & 16.8023 & 0.0008 \\
\hline 55079. & 16.8711 & 0.0011 \\
\hline 55102. & 16.8882 & 0.0019 \\
\hline 55309. & 16.9182 & 0.0008 \\
\hline 55321. & 16.9145 & 0.0009 \\
\hline 55363. & 16.8964 & 0.0008 \\
\hline 55415. & 16.8640 & 0.0011 \\
\hline 55447. & 16.8376 & 0.0009 \\
\hline 55753. & 16.3807 & 0.0007 \\
\hline 56030. & 15.6524 & 0.0009 \\
\hline 56075. & 15.5070 & 0.0009 \\
\hline 56084. & 15.4762 & 0.0017 \\
\hline 56132. & 15.3149 & 0.0012 \\
\hline 56186. & 15.1237 & 0.0009 \\
\hline 56380. & 14.3606 & 0.0008 \\
\hline 56460. & 14.0186 & 0.0012 \\
\hline 56482. & 13.9205 & 0.0008 \\
\hline 56755. & 12.6247 & 0.0019 \\
\hline 56774. & 12.5278 & 0.0008 \\
\hline 56830. & 12.2441 & 0.0009 \\
\hline 56856. & 12.1124 & 0.0008 \\
\hline 56920. & 11.7809 & 0.0007 \\
\hline
\end{tabular}

and small amplitudes (Eq. (26))

$\left|K_{1}\right|=0.89 \mathrm{~m} / \mathrm{s}$ and $\left|K_{2}\right|=0.18 \mathrm{~m} / \mathrm{s}$.

Since the precision of the instrument is supposed to be around $0.8 \mathrm{~m} / \mathrm{s}$, it should be able to detect the signal from $K_{1}$, but not from $K_{2}$.

With the 50 data points listed in Table 2 we will now apply the traditional techniques used to search for planets. The orbit of the binary CM introduces large amplitude variations in the radial velocity of the G-star and at first glance we can only detect these variations. Using the iterative Levenberg-Marquardt 


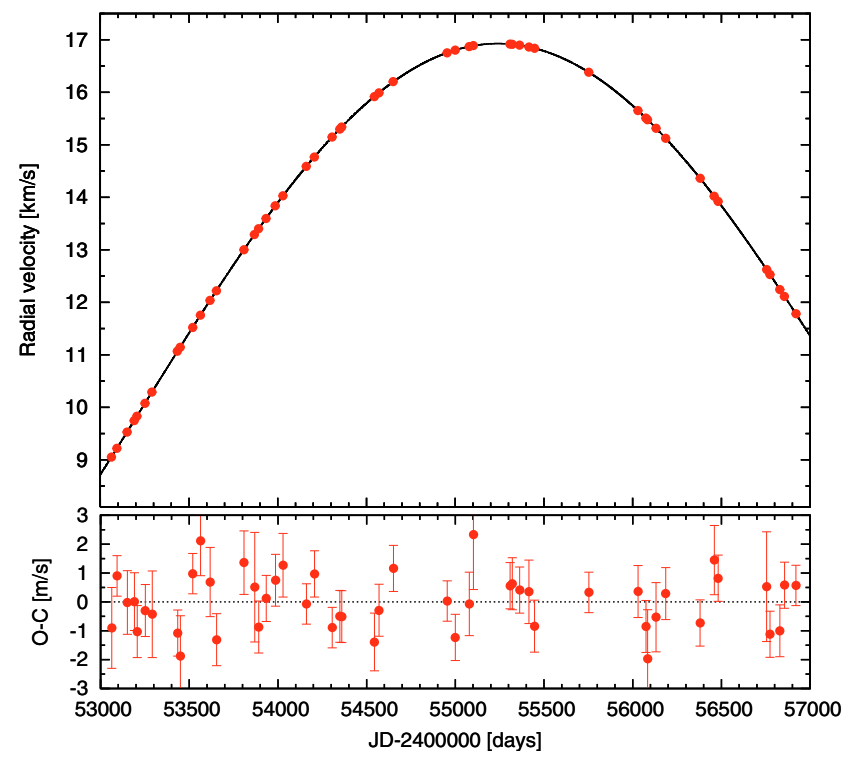

Fig. 4. Radial velocities for the G-star superimposed on a Keplerian orbital solution due to the close binary CM. The remaining residuals are slightly above the precision of the instrument and the observer can suspect of the existence of second companion in the system.

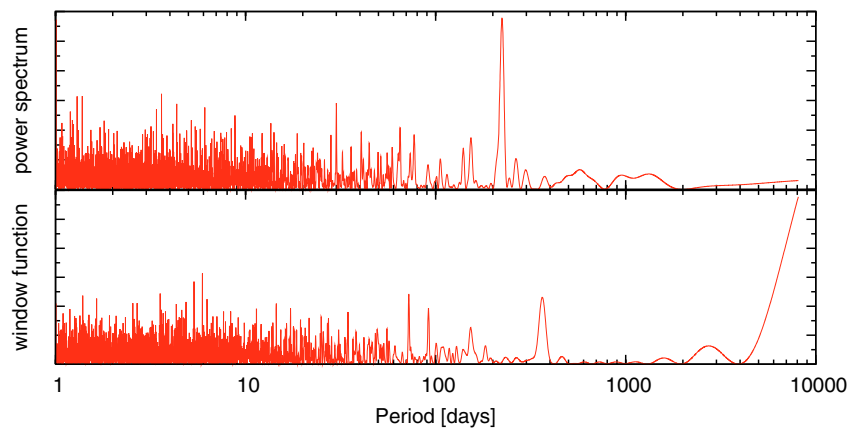

Fig. 5. Frequency analysis (top) and periodogram of the dates (bottom) for residual radial velocities when the contribution from the binary $\mathrm{CM}$ is subtracted. An important peak is detected at $P=223$ day, which can be interpreted as a second body in the system.

method (Press et al. 1992), we then first attempt to fit the complete set of radial velocities with a single orbiting companion in a Keplerian orbit. This fit yields a stellar companion with $P=$ 8010 day, $e=0.002$ and a minimum mass of $1.044 M_{\odot}$ (Fig. 4). These parameters correspond very well to the orbit of the G-star around the binary's CM. The mass determined by the fit is the total mass of the close binary system, $M=M_{1}+M_{2}=1.05 M_{\odot}$.

In Figure 4 we plot the radial velocity of the star superimposed on the binary $\mathrm{CM}$ orbit and also the velocity residuals after subtracting these long term variations. The precision of the instrument is $\sim 1 \mathrm{~m} / \mathrm{s}$, but the residuals, although very small, show amplitude variations that can reach almost $\pm 3 \mathrm{~m} / \mathrm{s}$. A trained observer can then suspect for the existence of something else in the data. To check it in a simple way we perform a frequency analysis of the residuals (Fig. 5). We can then see an important peak around 223 day, clearly indicating that something else is hidden in the data. We also plot a periodogram of the data, and since there is no peak at the same frequency (there is only a small one around 364 day) we conclude that the signal is true.

We then fit the radial velocities (Table 2) using a model with two Keplerian orbits. It yields for the planetary companion $P_{1}=222.6$ day, $e=0.049$ and a minimum mass of $20.6 M_{\oplus}$,
Table 3. Orbital parameters for two bodies orbiting the G-star, obtained with a two-Keplerian fit to the observational data.

\begin{tabular}{llcc}
\hline \hline Param. & [Unit] & Planet & Binary CM \\
\hline$V_{0}$ & {$\left[\mathrm{~km} \mathrm{~s}^{-1}\right]$} & \multicolumn{2}{c}{$10.021 \pm 0.037$} \\
$P$ & {$[\mathrm{day}]$} & $222.61 \pm 1.22$ & $8018.0 \pm 32.5$ \\
$e$ & & $0.0486 \pm 0.1725$ & $0.0016 \pm 0.0002$ \\
$\omega$ & {$[\mathrm{deg}]$} & $94.04 \pm 237.2$ & $280.35 \pm 34.93$ \\
$K$ & {$[\mathrm{~m} / \mathrm{s}]$} & $1.07 \pm 0.19$ & $6904.6 \pm 30.1$ \\
$T$ & {$[\mathrm{JD}-2400000]$} & $53839.0 \pm 146.9$ & $53471.9 \pm 778.0$ \\
\hline$a_{1} \sin I$ & {$\left[10^{-3} \mathrm{AU}\right]$} & 0.022 & 5089 \\
$f(m)$ & {$\left[10^{-3} M_{\odot}\right]$} & $2.8 \times 10^{-11}$ & 273.5 \\
$m_{2} \sin I$ & {$\left[M_{\oplus}\right]$} & 20.6 & $3.5 \times 10^{5}$ \\
$m_{2} \sin I$ & {$\left[M_{\odot}\right]$} & $6.2 \times 10^{-5}$ & 1.046 \\
$a$ & {$[\mathrm{AU}]$} & 0.719 & 9.95 \\
\hline $\mathrm{rms}$ & {$[\mathrm{m} / \mathrm{s}]$} & \multicolumn{3}{c}{0.56} \\
$\sqrt{\chi^{2}}$ & & \multicolumn{3}{c}{0.51} \\
\hline
\end{tabular}

Errors are given by the standard deviation $\sigma$. The orbital period of the outer body is longer than the data acquired and thus we are unable to complete constrain its orbit. The eccentricity and the periastron of the inner planet are also very badly constrained because we are at the limit of the instrument resolution. Despite all the uncertainties, the adjusted parameters for the planet agree with our model predictions (Sect. 3.5).

while for the binary's CM we get approximately the same orbital parameters as before (Table 3). Despite all the uncertainties in the orbital parameters, the use of a second Keplerian orbit is a better approximation than the single Keplerian model, since the velocity residuals drop to $\mathrm{rms}=0.56 \mathrm{~m} / \mathrm{s}$, below the precision of the instrument (they were above $1 \mathrm{~m} / \mathrm{s}$ before).

In Figure 6 we plot the phase-folded residual radial velocities when the contribution of the binary CM is subtracted. The data is superimposed on the orbital solution of the planetary companion, which really looks like a planet. The remaining $\mathrm{O}-\mathrm{C}$ is below the precision of the instrument $(\sim 1 \mathrm{~m} / \mathrm{s})$ and therefore we do not find any additional signal in the data. This is confirmed when we perform a frequency analysis of these residuals: no relevant peak is found, and there is no signal around $P_{2}=211$ day, the period for which we expected to see the $n_{2}$ frequency (Eq. (35)).

By comparing the numerical determination period of the planet, $P_{1}=222.61 \pm 1.22$ day (Table 3 ) with its theoretical estimation $P_{1}=222.64$ day (Eq. (35)), we see that our prediction was correct. Results for the amplitude are not so good, but still exact since the numerical determination $K_{1}=1.07 \pm 0.19 \mathrm{~m} / \mathrm{s}$ (Table 3) includes within the error bar the theoretical estimation $K_{1}=0.89 \mathrm{~m} / \mathrm{s}$ (Eq. (36)). A better determination could be achieved with identical instrumental precision, but using a larger amount of data points. On the other hand, the amplitude $K_{2}=0.18 \mathrm{~m} / \mathrm{s}$ (Eq. (36)) is lower than $\sigma_{K_{1}}=0.19 \mathrm{~m} / \mathrm{s}$ (Table 3), the error bar of $K_{1}$, so there is no hope of determining it with the present experience.

From the numerical values of $n_{1}$ and $K_{1}$ we can infer the orbital parameters of the planet, $a_{\mathrm{p}}=0.719 \pm 0.003 \mathrm{AU}$ and $M_{\mathrm{p}}=$ $20.6 \pm 3.7 M_{\oplus}$, while the theoretical values were respectively $a_{\mathrm{p}}=0.719 \mathrm{AU}$ (Eq. (29)) and $M_{\mathrm{p}}=17.3 M_{\oplus}$ (Eq. (30)). The mass is overestimated because the amplitude $K_{1}$ was so.

Now that we know that a binary can mimic a planet, whenever we suspect that a binary can be responsible for the observed stellar wobble, we should also provide its parameters. From the values of $n_{1}$ and $\Omega$ (Table 3 ) we can obtain (Eq. (27))

$$
\frac{2 \pi}{\omega}=411.0 \pm 2.2 \text { day and } \frac{2 \pi}{\Omega}=8018 \pm 33 \text { day }
$$


Table 4. Model predictions for different examples of binary systems around a star with $M_{\star}=M_{\odot}$.

\begin{tabular}{ccccccccccccc}
\hline \hline & \multicolumn{4}{c}{ Binary system } & \multicolumn{4}{c}{ Frequencies } & \multicolumn{3}{c}{ Amplitudes } & \multicolumn{3}{c}{ Planet } \\
Ex. & $M_{1}$ & $M_{2}$ & $a$ & $a_{\mathrm{b}}$ & $\alpha$ & $2 \pi / \Omega$ & $2 \pi / n_{1}$ & $2 \pi / n_{2}$ & $\left|K_{1}\right|$ & $\left|K_{2}\right|$ & $M_{\mathrm{p}}$ & $a_{\mathrm{p}}$ \\
& $\left(M_{\odot}\right)$ & $\left(M_{\odot}\right)$ & $(\mathrm{AU})$ & $(\mathrm{AU})$ & & $(\mathrm{yr})$ & $(\mathrm{day})$ & $(\mathrm{day})$ & $(\mathrm{m} / \mathrm{s})$ & $(\mathrm{m} / \mathrm{s})$ & $\left(M_{\oplus}\right)$ & $(\mathrm{AU})$ \\
\hline 1 & 1.00 & 1.00 & 10.0 & 1.00 & 0.114 & 18.26 & 137.1 & 131.7 & 0.987 & 0.197 & 23.90 & 0.520 \\
2 & 1.00 & 1.00 & 10.0 & 1.50 & 0.172 & 18.26 & 265.6 & 246.0 & 4.081 & 0.816 & 123.1 & 0.809 \\
3 & 0.70 & 0.35 & 10.0 & 1.10 & 0.156 & 22.09 & 222.6 & 211.0 & 0.888 & 0.178 & 17.26 & 0.719 \\
4 & 1.00 & 0.10 & 10.0 & 1.50 & 0.210 & 21.82 & 363.7 & 333.3 & 1.000 & 0.200 & 23.47 & 0.997 \\
5 & 1.00 & 0.01 & 10.0 & 1.50 & 0.215 & 22.30 & 380.6 & 348.1 & 0.114 & 0.023 & 2.59 & 1.028 \\
6 & 1.00 & $10^{-3}$ & 10.0 & 1.50 & 0.216 & 22.36 & 382.5 & 349.7 & 0.012 & 0.002 & 0.26 & 1.031 \\
7 & $10^{-3}$ & $10^{-3}$ & 1.00 & 0.01 & 0.114 & 1.00 & 4.23 & 4.13 & $10^{-5}$ & $10^{-6}$ & $10^{-4}$ & 0.051 \\
8 & $10^{-3}$ & $10^{-6}$ & 1.00 & 0.01 & 0.144 & 1.00 & 6.06 & 5.86 & $10^{-7}$ & $10^{-8}$ & $10^{-7}$ & 0.065 \\
\hline
\end{tabular}
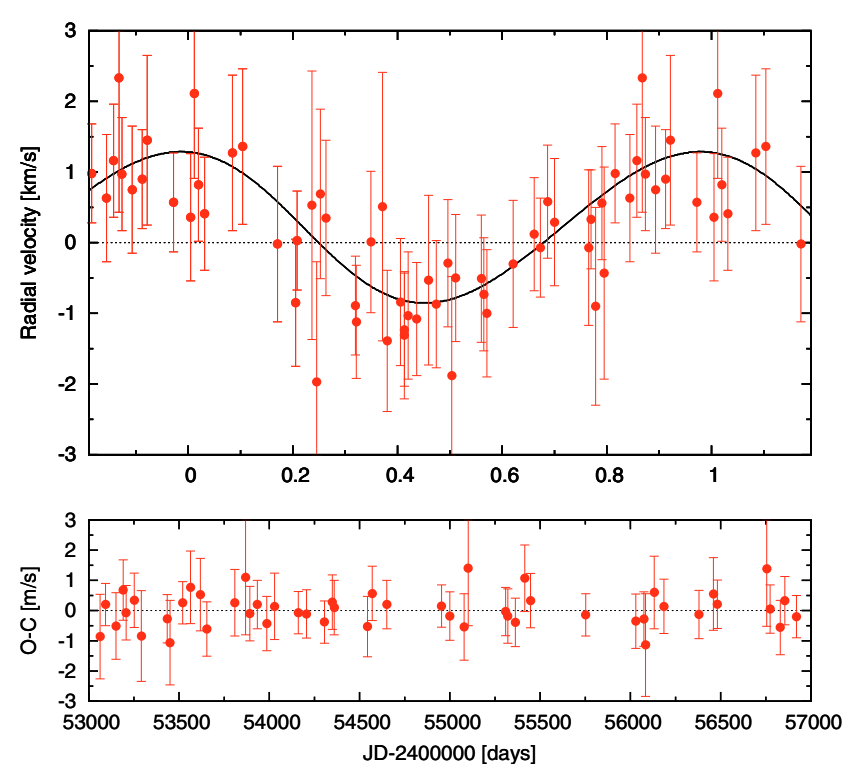

Fig. 6. Phase-folded residual radial velocities when the contribution of the binary $\mathrm{CM}$ is subtracted. Data is superimposed on a Keplerian solution with $P=222.6$ day and $e \approx 0$. The respective residuals as a function of Julian Date are displayed in the lower panel. We see that the remaining $O-C$ is below the precision of the instrument $(\sim 1 \mathrm{~m} / \mathrm{s})$ and therefore we do not expect to find any additional signal in the data.

which are in very good agreement with the true values (Eq. (34)). The individual masses of the stars in the close binary can also be resolved as well as the semi-major axis. Directly from the fit we have $a=9.95 \pm 0.03 \mathrm{AU}$ and $M=1.046 \pm 0.005 M_{\odot}$ (Table 3). Then, using Eqs. (32) and (33) we can respectively obtain

$a_{\mathrm{b}}=1.098 \pm 0.006 \mathrm{AU}$ and $\mu=0.275 \pm 0.062 M_{\odot}$.

By comparing the above determinations with the true parameters chosen for this system (Ex.\#3, Table 4) we verify that they match quite well. We thus conclude that a two-Keplerian approach of the stellar radial velocity is enough to determine the parameters of the triple system, i.e., we can keep the current software that is used to fit planets to the stellar data and do not need to develop a specific algorithm for binaries. Of course this in only true if we keep a Keplerian approach, the use of a $N$-body algorithm will give different results in the case of a planet or a binary.

\subsection{Different kinds of binary systems}

Now that we have seen that our formulae are correct we will apply them to a wide variety of binary systems' configurations. In Table 4 we see the predictions of the model for several binary systems around a star with $M_{\star}=M_{\odot}$. We chose only stable binary systems with $\alpha \ll 0.5$ (see Eq. (10)).

The first and second examples are the cases studied in Schneider \& Cabrera (2006): the star is being perturbed by close equal masses' binaries composed of stars with $1 M_{\odot}$. In Ex.\#1, $K_{1}$ is at the current resolution level $(\sim 1 \mathrm{~m} / \mathrm{s})$ while $K_{2}$ is 5 times smaller. In this case we may be lead to believe that the star has a planet companion with 1.4 Neptune masses. On the other hand, in Ex.\#2 we should be able to detect both $K_{1}$ and $K_{2}$; hence in this case we can conclude that the star's wobble is caused by a binary and not a planet companion.

The third example is the case discussed in the previous section. Here we have a triple system composed of stars with different spectral types. As we saw previously, in this case we can detect the peak with amplitude $K_{1}$, but not the one with amplitude $K_{2}$, and we are lead to believe that the star has a planet companion with about one Neptune mass.

In the fourth example the star is being perturbed by a close hierarchical binary composed of a star with $1 M_{\odot}$ and another body with $0.1 M_{\odot}$. We have $K_{1}$ at the current resolution level while $K_{2}$ is 5 times smaller. Again we are lead to believe that the star has a planet companion with 1.4 Neptune masses.

In the next two examples the binaries are composed of a star with $1 M_{\odot}$ and a brown-dwarf with $10 M_{J}$ (Ex.\#5) or a planet with $1 M_{J}$ (Ex.\#6). In these cases both peaks are currently undetectable $(\sim \mathrm{cm} / \mathrm{s})$ but could be within reach of future observation campaingns like CODEX (Pasquini et al. 2008).

Finally, in the last two examples we have a $1 M_{\odot}$ star and a binary composed of two Jupiter-sized planets (Ex.\#7) and a Jupiter-sized planet and an Earth-sized "satellite" (Ex.\#8). In both cases, the inferred values for $M_{\mathrm{p}}$ are too low for planets.

\section{Discussion and conclusion}

In this paper we studied a triple system composed of a star which we observe and a binary system. Our goal was to decide if the effect of the binary system on the star's center of mass motion could be mistaken as a planet companion to it.

Previous work done by Schneider \& Cabrera (2006) suggested that the effect of a star-star system could mimic the presence of a planet companion to the observed star. However, we found that this is not correct, since a more careful analysis of the observational data should allow us to distinguish between the two effects. Indeed, when there is a close binary system, the Fourier spectrum of the star's radial velocity should exhibit not one but two peaks located at nearby frequencies. This could also indicate the presence of two planets on close orbits, but since these systems are most likely unstable we should be able to reject this hypothesis. 
Nevertheless, we saw that it is sometimes possible to mistake (at least temporarily) the effect of a binary system by a planet. This happens when the observations are near the resolution limit so that we can identify the primary peak but not the secondary peak. However, even in this case our estimate for the fake planet's mass differs from that obtained by Schneider \& Cabrera (2006).

In this article we obtained tools for distinguishing between the two effects: we estimate the parameters of the binary system that could be mistaken by a planet and check if they are realistic. We recommend that, in future discoveries of planets around stars in binary systems, these formulas should be used in order to exclude the possibility of a false alarm.

We also wanted to evaluate the effect of different types of binary systems on the star's CM motion. In particular, we were interested in star-star, star-planet and planet-satellite systems. We saw that the effect of a star-star system is only significant when this is close to the observed star. For instance, a binary system of dwarf stars at $1 \mathrm{AU}$ from each other and at the distance $10 \mathrm{AU}$ from the observed star causes a wobble on the latter which would be at the current resolution level. We also saw that the effect of a star-planet system is currently undetectable although it is possibly within reach of future observation campaigns. Finally, we saw that the effect of a planet-satellite system is insignificant and should never be mistaken by a planet companion to the observed star as the implied mass would simply be too low.

Our conclusions were established for a triple system of bodies evolving in co-planar and circular orbits. However, it is known that these kind of systems may present eccentric orbits and different mutual inclinations. The inclusion of these two effects may change some of the results presented here.

Acknowledgements. M.H.M. Morais acknowledges financial support from FCTPortugal (grant SFRH/BPD/19155/2004).

\section{Appendix A:}

We re-write the equation of motion of the star $M_{\star}$ (Eq. (19)) using Cartesian coordinates in the $(\hat{\boldsymbol{l}}, \hat{\boldsymbol{J}})$ frame (see Fig. 2 ) such that

$\hat{\boldsymbol{r}}=\cos \theta \hat{\boldsymbol{\imath}}+\sin \theta \hat{\boldsymbol{\jmath}}$,

$\hat{\boldsymbol{u}}=-\sin \theta \hat{\boldsymbol{\imath}}+\cos \theta \hat{\boldsymbol{\jmath}}$,

with

$\theta=\theta_{0}+\Omega t$,

where $\theta_{0}$ is an initial phase (Fig. 2).

Thus we obtain

$$
\begin{aligned}
\ddot{X}= & -\frac{G M}{a^{2}}\left[\left(1+\frac{3}{4} \rho^{2} \frac{\mu}{M}\right) \cos \theta\right. \\
& \left.+\frac{9}{4} \rho^{2} \frac{\mu}{M} \cos 2 S \cos \theta+\frac{3}{2} \rho^{2} \frac{\mu}{M} \sin 2 S \sin \theta\right], \\
\ddot{Y} & =-\frac{G M}{a^{2}}\left[\left(1+\frac{3}{4} \rho^{2} \frac{\mu}{M}\right) \sin \theta\right. \\
& \left.+\frac{9}{4} \rho^{2} \frac{\mu}{M} \cos 2 S \sin \theta-\frac{3}{2} \rho^{2} \frac{\mu}{M} \sin 2 S \cos \theta\right],
\end{aligned}
$$

where $\rho=a_{b} / a \ll 1$.

In order to solve this set of differential equations we perform the transformation of coordinates

$$
\left(\begin{array}{c}
\bar{X} \\
\bar{Y}
\end{array}\right)=\left(\begin{array}{cc}
\sin \theta & -\cos \theta \\
\cos \theta & \sin \theta
\end{array}\right)\left(\begin{array}{c}
X-a_{\star} \cos \theta \\
Y-a_{\star} \sin \theta
\end{array}\right),
$$

with

$a_{\star}=\frac{M}{M+M_{\star}}\left(1+\frac{3}{4} \rho^{2} \frac{\mu}{M}\right) a$.

The set of differential equations becomes

$\ddot{\bar{X}}-2 \Omega \dot{\bar{Y}}-\Omega^{2} \bar{X}=-12 \omega^{2} \delta \sin 2 S$,

$\ddot{\bar{Y}}+2 \Omega \dot{\bar{X}}-\Omega^{2} \bar{Y}=-18 \omega^{2} \delta \cos 2 S$,

with

$\delta=\frac{\mu}{8 M}\left(\frac{a_{\mathrm{b}}}{a}\right)^{4} a$.

This admits the solution

$\bar{X}=\delta_{X} \sin 2 S$,

$\bar{Y}=\delta_{Y} \cos 2 S$,

where

$$
\begin{aligned}
-4(\omega-\Omega)^{2} \delta_{X}+4 \Omega(\omega-\Omega) \delta_{Y}-\Omega^{2} \delta_{X} & =-12 \omega^{2} \delta, \\
-4(\omega-\Omega)^{2} \delta_{Y}+4 \Omega(\omega-\Omega) \delta_{X}-\Omega^{2} \delta_{Y} & =-18 \omega^{2} \delta .
\end{aligned}
$$

The solution to Eqs. (A.13) and (A.14) is then

$\delta_{X}=12 \delta \frac{4(1-\beta)^{2}+\beta^{2}+6 \beta(1-\beta)}{\left(4(1-\beta)^{2}-\beta^{2}\right)^{2}}$
$\delta_{Y}=12 \delta \frac{6(1-\beta)^{2}+3 \beta^{2} / 2+4 \beta(1-\beta)}{\left(4(1-\beta)^{2}-\beta^{2}\right)^{2}}$,

with $\beta=\Omega / \omega \ll 1$.

To zero order in $\beta$,

$\delta_{X}=3 \delta$,

$\delta_{Y}=4.5 \delta$,

hence

$\left(\begin{array}{c}X \\ Y\end{array}\right)=\left(\begin{array}{l}a_{\star} \cos \theta \\ a_{\star} \sin \theta\end{array}\right)+\left(\begin{array}{cc}\sin \theta & \cos \theta \\ -\cos \theta & \sin \theta\end{array}\right)\left(\begin{array}{c}3 \delta \sin 2 S \\ 4.5 \delta \cos 2 S\end{array}\right)$.

Note that this does not match the solution obtained in Schneider \& Cabrera (2006):

- The 2 nd term in Eq. (A.19) is rotated by an angle $\theta$.

- The frequency of the 2 nd term is $2(\omega-\Omega) \approx 2 \omega$ as we assume $\Omega \ll \omega$.

- The expressions for $\Omega$ (Eq. (14)) and $a_{\star}$ (Eq. (A.7)) take into account the mass $M_{\star}$.

\section{References}

Correia, A. C. M., Udry, S., Mayor, M., et al. 2008, A\&A, 479, 271

Henrard, J. 1979, Celestial Mechanics, 19, 337

Hilditch, R. W. 2001, An Introduction to Close Binary Stars (Cambridge University Press)

Lagrange, A.-M., Beust, H., Udry, S., Chauvin, G., \& Mayor, M. 2006, A\&A, 459,955

Markellos, V. V., \& Roy, A. E. 1981, Celestial Mechanics, 23, 269

Mayor, M., Pepe, F., Queloz, D., et al. 2003, The Messenger, 114, 20

Mayor, M., Udry, S., Naef, D., et al. 2004, A\&A, 415, 391

Murray, C. D., \& Dermott, S. F. 1999, Solar System Dynamics (Cambridge University Press)

Neuhäuser, R., Mugrauer, M., Fukagawa, M., Torres, G., \& Schmidt, T. 2007, A\&A, 462, 777

Pasquini, L., Avila, G., Delabre, B., et al. 2008, in Precision Spectroscopy in Astrophysics, ed. N. C. Santos, L. Pasquini, A. C. M. Correia, \& M. Romaniello, 249

Press, W. H., Teukolsky, S. A., Vetterling, W. T., \& Flannery, B. P. 1992, Numerical recipes in FORTRAN. The art of scientific computing (Cambridge: University Press), 2nd edn

Queloz, D., Mayor, M., Weber, L., et al. 2000, A\&A, 354, 99

Schneider, J., \& Cabrera, J. 2006, A\&A, 445, 1159

Zucker, S., Mazeh, T., Santos, N. C., Udry, S., \& Mayor, M. 2004, A\&A, 426, 695 